Voix et Images

\title{
Distance, point de vue, voix et idéologie dans les Fous de Bassan d'Anne Hébert
}

Neil B. Bishop

Volume 9, numéro 2, hiver 1984

Roland Giguère

URI : https://id.erudit.org/iderudit/200443ar

DOI : https://doi.org/10.7202/200443ar

Aller au sommaire du numéro

Éditeur(s)

Université du Québec à Montréal

ISSN

0318-9201 (imprimé)

1705-933X (numérique)

Découvrir la revue

\section{Citer cet article}

Bishop, N. B. (1984). Distance, point de vue, voix et idéologie dans les Fous de Bassan d'Anne Hébert. Voix et Images, 9(2), 113-129.

https://doi.org/10.7202/200443ar d'utilisation que vous pouvez consulter en ligne.

https://apropos.erudit.org/fr/usagers/politique-dutilisation/ 


\title{
Distance, point de vue, voix et idéologie dans les Fous de Bassan d'Anne Hébert
}

\author{
par Neil B. Bishop, Université de Victoria
}

\section{Pour Annick}

Une grande praticienne de la narration littéraire, du récit de fiction: c'est ainsi que la critique s'accorde pour voir Anne Hébert, surtout depuis Kamouraska. La présente étude vise à analyser certains aspects de la structure narrative du roman le plus récent d'Anne Hébert, les Fous de Bassan. ${ }^{1}$ Choisis en raison de leur importance décisive dans les économies narrative et signifiante du roman, ${ }^{2}$ ces aspects se nomment distance, point de vue et voix, termes évocateurs de réalités textuelles et que nous utiliserons avec le sens que leur donne Gérard Genette dans son ouvrage Figures $I I I .{ }^{3}$ Ces aspects narratifs permettront de découvrir dans ce roman une substance idéologique de caractère féministe.

Nous serons amenés à préciser nos assises théoriques, mais il convient d'effectuer d'abord une description d'ensemble de l'objet de notre étude, le roman les Fous de Bassan, avant de l'analyser en fonction des trois aspects identifiés ci-dessus. Ce roman comporte six parties, comprenant chacune une page de titre, d'une longueur variant entre vingt-deux et cinquante-huit pages. Une présentation liminaire de ces parties et des références temporelles qui les accompagnent dans la table des matières rendra notre analyse plus aisée à suivre: 4

1. Anne Hébert, les Fous de Bassan (roman), Paris, Ed. du Seuil, 1982, 253 p. Désormais nous n'indiquerons que les références paginales dans notre texte.

2. «les économies narrative et signifiante»: en effet, ici les deux vont de pair, les structures narratives étudiées - distance, point de vue et voix - informent, notre étude tente de le démontrer, le sens romanesque.

3. Gérard Genette, Figures III, Paris, Ed. du Seuil, 1972, pp. 183-267.

4. À l'encontre de ce qu'affirme la table des matières le premier «livre» commence, non à la page 9 , mais à la page 11. À la page 9 figure un «Avis au lecteur " qui insiste sur le caractère fictif du contenu diégétique. 
Le livre du révérend Nicolas Jones

automne 1982

Lettres de Stevens Brown à Michel Hotchkiss

été 1936

Le livre de Nora Atkins

été 1936

Le livre de Perceval Brown et de quelques autres

été 1936

Olivia de la Haute Mer

sans date

Dernière lettre de Stevens Brown à Michel Hotchkiss

automne 1982.

Cette table des matières fait ressortir certaines caractéristiques pertinentes du roman:

1. une pluralité des narrateurs (il en sera souvent question dans cette étude);

2. une pluralité des temps.

Mais de combien d'époques s'agit-il? La table des matières insiste sur deux époques: automne 1982, été 1936. Celle-ci est la plus importante du roman; l'autre époque importante, automne 1982, correspond non à l'époque de l'écritures mais à celle de la publication du roman, ce qui rend ce dernier d'autant plus captivant, puisque cet aspect du temps romanesque permet au lecteur de l'automne 1982 de mieux s'identifier aux consciences narrantes de la première et de la dernière partie du roman; identification qui, une fois instaurée dès la première partie, tend à se maintenir tout au long du roman. Cette tendance à l'identification lecteur-conscience narrante constitue une illusion - le lecteur a presque l'impression que c'est lui qui écrit le roman et est conforté dans cette illusion par l'emploi prédominant de la narration à la première personne - qui renforce le caractère mimétique du roman. Il s'agit là d'un premier contact avec la problématique de la distance romanesque: on sait que le mimétique est moins distant que le diégétique. ${ }^{6}$

La table des matières exige que l'on interroge ce geste scriptural consistant à donner, après le titre de la cinquième partie, une référence temporelle tout ce qu'il y a de plus vague: «sans date». En fait, le livre d' «Olivia de la Haute Mer» relève d'une a-temporalité, ou éternité, puisqu'il s'agit du livre (ainsi que du point de vue et de la voix) d'une morte - nous y reviendrons.

5. Des textes publiés en septembre 1982 ne pouvant avoir été écrits à l'automne 1982. A. Hébert a d'ailleurs précisé avoir commencé à écrire les Fous de Bassan en 1977 (entretien avec Jean Royer, le Devoir, 11 décembre 1982, p. 21).

6. G. Genette, op. cit., p. 184. 
D'autres traits pertinents à inclure dans cette description d'ensemble du roman sont:

1. Une structure temporelle encore plus complexe que ne l'indiquent les références temporelles de la table des matières. Malgré la précision «automne 1982», la première partie se préoccupe surtout de l'été 1936; comme d'autres elle évoque parfois des moments antérieurs à cet été-là. Si Olivia évoque parfois son enfance (pp. 205-212, 213), Nora évoque un avenir virtuel et optatif («Un jour ce sera l'amour fou», "Nous serons mari et femme, roi et reine, pour l'éternité», p. 120). L'importance des événements du soir du 31 août 1936 fera de cette date un moment privilégié, objet de nombreuses analepses et prolepses ainsi que d'une bonne partie des livres de plusieurs personnages. Une part importante du «livre de Perceval Brown et de quelques autres" raconte l'enquête policière survenue au cours des semaines qui suivirent la disparition, le 31 août 1936, de Nora et d'Olivia.

2. Une pluralité des récits. Celle-ci se manifeste dès «Le livre du révérend Nicolas Jones» qui raconte aussi bien le présent du narrateur que l'été 1936, avec des références à d'autres époques (l'enfance du narrateur, par exemple). L'histoire des événements de l'été 1936 revêt ainsi un statut métadiégétique (récit second) par rapport aux événements du présent de la narration (tout comme le récit du passé d'Élisabeth d'Aulnières/Tassy par rapport au présent d'Élisabeth Rolland, comme l'a bien montré Robert Harvey. ${ }^{7}$ De même, le livre d'Olivia de la Haute Mer fait alterner le récit du passé (enfance d'Olivia, été 1936, soir du 31 août 1936) et celui de sa «vie» présente («vie» a-temporelle, admirablement fictive, comme il convient à une morte, et relevant plus de la description et de la poésie que de la narration). Comme dans Kamouraska et les Enfants du Sabbat (passé enfantin dans la montagne de B..., présent de la vie au couvent), la pluralité temporelle romanesque dans les Fous de Bassan a partie liée avec une pluralité des récits, d'où une polyvalence narrative. Ces deux pluralités renvoient à une troisième, celle des narrateurs, chacune des six parties du roman étant le (voire les) récit(s) d'un personnage-narrateur (qui a aussi un statut d'actant dans le ou les récit(s) qu'il narre) racontant de son point de vue une époque, des personnages et des événements racontés ailleurs, le plus souvent, du point de vue d'un autre personnage-narrateur (et, donc, par une autre voix).

3. Le caractère autodiégétique des différentes parties du roman (et, donc, du récit de chaque personnage-narrateur), chaque récit étant le plus souvent raconté à la première personne du singulier. Il faudra revenir, toutefois, sur l'emploi occasionnel de pronoms autres que le «je». .

Ces cinq traits du roman dans son ensemble permettent de préciser les assises théoriques et méthodologiques de notre étude. Il est clair que nous

7. Robert Harvey, «Kamouraska» d'Anne Hébert: une écriture de la Passion, suivi de Pour un nouveau "Torrent», Montréal, Hurtubise HMH, Cahiers du Québec, collection Littérature, 1982, pp. 11, 39-44. 
aurons surtout affaire au(x) narrateur(s). La problématique de la distance évolue principalement en fonction de la distance à laquelle se tient - ou cherche à se tenir - tel ou tel narrateur par rapport aux funestes événements de l'été 1936 et notamment du 31 août 1936. D'autre part, le caractère autodiégétique des six parties du roman simplifie la double problématique du point de vue et de la voix, celle, selon la formulation genettienne, de savoir «qui voit?» et «qui parle?»: ${ }^{8}$ sous l'effet de l'autodiégétique, les deux "qui» tendent à fusionner, à relever du narrateur, ou du personnagenarrateur-actant. En effet, «La seule focalisation logiquement impliquée par le récit 'à la première personne' est la focalisation sur le narrateur».9 Nous pourrons ainsi effectuer simultanément, le plus souvent, l'analyse des problématiques du point de vue et de la voix dans les Fous de Bassan, quitte à étudier ensuite quelques aspects de celle-ci qui exigent un traitement à part.

Les habitants de Griffin Creek, descendants de Loyalistes, sont protestants et puritains. Le refoulé, dans cet univers, ne tarde pas à se révéler comme étant le désir sexuel (Stevens Brown dira même, dans sa dernière lettre, qui termine le roman, que «Tout le mal vient de là», p. 242): l'on comprend dès lors que Nicolas Jones, narrateur, se remémorant cette soirée du «barn dance» au cours de laquelle il n'arrivait plus à cacher son désir pour ses jeunes nièces, tentera de se dissocier de ce moment de péché, d'accroître la distance entre lui-même et l'actant-pécheur qu'il fut alors, en abandonnant subitement le «je» pour parler de lui-même à la troisième personne:

Mon Dieu est-ce possible? Dois-je revivre à l'instant l'été 1936, être à nouveau celui qui convoite la vie et se fait complice de la mort?

Le soir du barn dance Nicolas Jones danse avec les petites Atkins, les fait tourner et virevolter à tour de rôle, les tient par la main et par la taille, respire leur odeur à plein nez, ivre sans avoir bu une goutte d'alcool (...) (p. 46).

Quand sa femme, consciente du désir extra-conjugal (et incestueux) qu'il éprouve, se lève pour aller se suicider, à nouveau le narrateur Nicolas Jones tente de mettre le plus de distance possible entre lui et toute responsabilité dans ce drame en n'avouant les faits et sa propre inaction qu'à la troisième personne: «son mari le pasteur ne s'est pas retourné dans son sommeil, n'a pas remarqué la place vide dans le grand lit» (p. 49). La pertinence du critère de la distance narrative est soulignée par Nicolas Jones lui-même qui précise la visée de sa fonction de régie face à son propre récit:

M'enfoncer dans mon fauteuil. Prendre mes distances. Ne plus être hors de moi, debout à la frontière de la terre et de l'eau comme une croix du chemin sur laquelle déferlent le vent et toute la vie ancienne (...) Que passe au loin l'homme de trente-cinq ans (p. 41; nous soulignons).

8. G. Genette, op. cit., p. 203

9. Ibid., p. 219. 
Vers la fin de son «livre», ce même narrateur tente de se distancier de lui-même tel qu'il est par l'emploi de la troisième personne («Le temps qu'il faut à un vieil homme pour s'habiller (...) criant pour qu'on lui apporte son col», p. 53), cette tentative de distanciation découlant ici, sans doute, d'une prise de conscience de l'échec de toute une existence, de l'hypocrisie et du vide qui y règnent depuis des décennies. Ces diverses tentatives de distanciation sont d'autant plus significatives que, le plus souvent, Nicolas Jones n'a aucun mal à narrer le récit de son passé à la première personne.

Chez Stevens Brown, assassin de Nora et d'Olivia, cette même volonté de mettre une distance entre soi et sa culpabilité prend la forme du désir de pouvoir parler de soi à la troisième personne, de troquer son identité d'assasin contre une identité moins culpabilisante:

Être quelqu'un d'autre. Ne plus être Stevens Brown. (...) Il n'est peutêtre pas trop tard pour changer de peau définitivement (...) Ne pas laisser la suite de mon histoire à Griffin Creek se dérouler jusqu'au bout (pp. 79-80).

Ce passage et plusieurs autres (cf. pp. 85-86) constituent des prolepses qui soulignent que l'écriture des Fous de Bassan a des buts tout autres que le réalisme (ces prolepses étant irréalistes et invraisemblables dans des lettres datées d'avant le 31 août 1936). En outre, l'on découvre ainsi que l'un des buts que vise cette écriture est la création et le maintien du suspense: par des prolepses qui en laissent deviner long, mais pas tout, sur le «bout» de l'«histoire», la vérité du 31 août 1936, cette écriture atteint son but.

Cet emploi des prolepses augmente la tension et donc l'intérêt romanesques en contrariant le désir de mettre une distance entre soi-même, narrateur, et le personnage-actant de l'été 1936 et en s'opposant aux techniques narratives par lesquelles des narrateurs tels que $\mathrm{N}$. Jones et $\mathrm{S}$. Brown tentent de susciter cette distance. Pour eux, et dans leur récit, la prolepse, c'est l'ennemie, tout comme Élisabeth Rolland, pendant longtemps, cherche à empêcher sa mémoire et son monologue intérieur de prendre le chemin de «Kamouraska, l'anse de Kamouraska» et le 31 janvier 1839.10

Si les prolepses et la volonté de les refuser (volonté que traduit le fait que ces prolepses ne disent pas tout sur l'aboutissement fatal de l'été 1936) provoquent un effet de suspense chez le lecteur, la distance entre Olivia de la Haute Mer, narratrice, et Olivia Atkins, personnage-actant, est, elle, fort émouvante. Olivia, plus que toute autre, plus même que sa cousine Nora (morte très rapidement) a souffert l'enfer du 31 août 1936, le viol et l'étranglement: comment s'étonner que, narratrice, «limpide sur la mer comme une larme» (p. 212), Olivia cherche souvent à maximiser la distance entre elle-même et ce funeste passé? Plus que tout autre personnage du roman Olivia parle d'elle-même à la troisième personne. Plus précisément, Olivia

10. Anne Hébert, Kamouraska (roman), Paris, Ed. du Seuil, 1970, p. 224. 
de la Haute Mer dira «elle» en parlant d'Olivia Atkins. Certes, son «livre» oscille entre le «elle» et le «je», un «je» parfois utilisé pour parler même d'Olivia Atkins, quand Olivia de la Haute Mer accepte, nous dira-t-elle, de «réintégrer mon nom comme une coquille vide. Reprendre le fil de ma vie» (p. 212). Ces oscillations traduisent l'attitude ambiguë d'Olivia envers Stevens:

Que Stevens se montre une fois encore (...) Qu'il me parle encore, qu'il me touche avec ses deux mains d'homme (...) Le voir. Être vue par lui (...) Exister encore une fois, éclairée par lui (...) devenir à nouveau matière lumineuse et vivante, sous son regard.

(...)

Quelque part (...) l'ordre de mort est donné. (...) Je n'avais qu'à fuir avant même que Stevens pose sur moi ses yeux d'enfant.

(...)

ne plus profiter de la marée pour retourner à Griffin Creek. (...) Non, non ce n'est pas moi, c'est le désir qui me tire et m'amène, chaque jour, sur la grève (pp. 220-221).

La cause de ces oscillations, c'est que Stevens Brown est pour Olivia de la Haute Mer à la fois son meurtrier répugnant et l'attirant objet de son désir. Si elle accepte parfois de revivre l'été 1936, si elle revient hanter Griffin Creek, c'est mue par la force du désir.

La distance, dans le livre d'Olivia, est créée à l'aide de deux procédés scripturaux. L'un, on l'a vu, est l'emploi de la troisième personne, surtout dans les passages qui évoquent les rencontres enfantines d'Olivia et de Stevens (p. 205, 213), comme si, pour qu'Olivia puisse éviter tout à fait de revivre son histoire avec Stevens, il avait fallu qu'ils ne se soient jamais connus, ou que la petite Olivia jouant avec Stevens soit restée une autre, ne soit point devenue le «je» d'Olivia Atkins l'adolescente, puis d'Olivia de la Haute Mer. L'autre procédé scriptural servant à mettre de la distance entre cette narratrice et la tragique diégèse de l'été 1936, c'est l'emploi de l'espace romanesque: pour s'éloigner de l'horreur du souvenir, Olivia quitte souvent la grève et prend le large. Pour éviter de rencontrer à nouveau Stevens sur son passage, un 31 août remémoré, Olivia devra fuir les lieux, interdire à sa mémoire un plus long séjour à Griffin Creek: «Je n'ai plus rien à faire ici (...) Regagnons la haute mer (...) je quitte la grève de mon enfance et les mémoires obscures de ma vie ancienne» (p. 204). Mais le désir, dira Olivia, «me tire et m'amène, chaque jour, sur la grève» (p. 221). S'instaure ainsi un va-et-vient, la distance entre la "haute mer» et Griffin Creek étant tantôt abolie par la puissance du désir, tantôt réclamée par une narratrice avide d'échapper à l'horreur finale:

Qui le premier parle de se rendre sur la grève?

Que je lève seulement la tête et je verrai son visage, la dureté des os ruisselants de lune. Ses lèvres se retroussent sur ses dents en un sourire étrange. Mon Dieu vais-je mourir à nouveau?

Je n'ai que juste le temps de (...) m'échapper sur la mer avant que ne revienne, dans toute sa furie, la soirée du 31 août 1936 (pp. 224-225). 
Cette utilisation de l'espace romanesque pour créer un effet de distance narrative est révélatrice, à son tour, de la science du récit hébertien.

Particulièrement enrichissante pour les phénomènes narratifs relevant du point de vue et de la voix est la multiplicité de narrateurs dont jouit les Fous de Bassan. Puisque «La seule focalisation logiquement impliquée par le récit 'à la première personne' est la focalisation sur le narrateur"," les Fous de Bassan, écrit principalement à la première personne, n'aurait pu nous offrir qu'un seul point de vue, n'était-ce de cette construction du roman en six «livres», chacun narré par son narrateur propre, chaque «je» étant «un autre». Dès lors, les Fous de Bassan offre toutes les ressources de la «focalisation multiple», "où le même événement peut être évoqué plusieurs fois selon le point de vue de plusieurs personnages». 12

Des événements, tantôt antérieurs, tantôt postérieurs au 31 août, sont présentés par des narrateurs différents, chacun les narrant tels qu'il les a vus et vécus, vision et expérience différentes de celles que communiquent au lecteur, à propos de ces mêmes événements, d'autres narrateurs. D'ailleurs, tel personnage-narrateur a vu et vécu tel événement de plus près qu'un autre personnage: celui-là donnera donc, sur l'événement, plus d'informations, et en "gros plan» par rapport à la vision qu'en donnera celui-ci. C'est dire que la problématique du point de vue implique celle de la distance. ${ }^{13}$ Cette interaction du point de vue et de la distance narrative se manifeste, par exemple, à travers l'évocation d'une tempête de trois jours et trois nuits survenue juste avant le 31 août 1936. Évoquée par Stevens Brown dans sa lettre en date - significativement - du 31 août 1936,14 cette tempête est racontée d'une autre façon par lui que par Nora et Olivia. Stevens, qui a tout fait pour vivre directement la tempête - métaphore de son désir et des violences auxquelles aboutit ce désir - en restant dehors nuit et jour, la raconte (pp. 100-104) avec plus de détails que ne le font Nora (pp. 131-133) et Olivia (pp. 221-222) dans leur livre respectif. Stevens donne de la tempête un récit qui tend à quitter la diégésis pour la mimésis et sa diminution caractéristique de la distance narrative; les récits plus brefs, moins mimétiques que font de cette même tempête Nora et Olivia, correspondent à ce que dit Genette du diégétique: «Indirection et condensation (rendent le 'récit pur') plus distant que l'imitation': il en dit moins, et de façon plus médiate».15

Comme les événements, les personnages sont vus et racontés par des narrateurs différents dont, souvent, le personnage concerné lui-même, mué

11. G. Genette, op. cit., p. 219.

12. Ibid., p. 207.

13. G. Genette rapproche distance et point de vue en tant que deux «modalités» du «mode» (ibid., pp. 183-184).

14. Une regrettable coquille dans notre exemplaire du roman fait lire « $1930 »$, p. 99 , mais son statut d'erreur ne laisse aucun doute, vu que toute la série de lettres date de l'été 1936 (voir pp. $55,57,253$ ).

15. G. Genette, op. cit., p. 184. 
en narrateur. En particulier, tel personnage que le lecteur n'avait vu que de l'extérieur, présenté par un autre, révélera, le moment venu de donner texte à son propre récit, une autre vision de lui-même et des rapports entre les deux personnages impliqués. À nouveau les rapports entre Stevens et Nora, d'une part, et, de l'autre, Stevens et Olivia se laissent mieux discerner et comprendre à l'aide des livres des deux jeunes filles. Stevens a une vision fort sexiste de Nora, la méprisant à cause du désir qu'elle éprouve pour lui (p. 90; sexiste à l'extrême est la vision des personnages féminins dans toutes les lettres de Stevens, nous y reviendrons...). Nora présente une autre vision des rapports possibles entre une fille et un garçon - «il serait si facile de s'entendre comme deux personnes, égales entre elles, dans l'égalité de leur désir» (p. 127). Olivia, comme Nora, confirme la force de son désir, désir qu'avait bien deviné Stevens. Celui-ci, toutefois, attribue à tort le comportement réservé d'Olivia envers lui à une certaine hypocrisie, à un refus de se reconnaître femme, avec un corps et un désir de femme. Olivia, on l'a vu, était bien consciente de son désir; son refus d'assouvir celui-ci avec Stevens provenait beaucoup plus d'une méfiance fondamentale, et comme héréditaire (voir les nombreuses références à ses mère et grand-mères l'avertissant du mal qui est en Stevens) envers ce sexiste qui n'a pour la femme que mépris, voire haine, et qui ira jusqu'à tuer la femme en tuant ses deux cousines.

La pluralité des narrateurs permet de mieux cerner et saisir cette «vie secrète des passions» dont parle Gabrielle Poulin, 16 la psychologie profonde des personnages, les mécanismes de la communication et de l'incommunicabilité. Cette même pluralité des points de vue contribue à l'effet de suspense, car elle peut avoir quelque chose du roman policier (cf. les techniques de l'interrogatoire et de l'interrogatoire contradictoire qu'utilisent les policiers en tentant de découvrir l'assassin de Nora et d'Olivia). La pluralité des points de vue met à l'épreuve la vérité des divers personnages en aménageant la possibilité de confronter leurs récits différents, leurs visions des événements et des êtres et des rapports entre eux. Est pertinentè à ce propos la remarque de $\mathbf{R}$. Harvey au sujet du récit second dans Kamouraska: «Aussi, le principal intérêt de ce récit parallèle tient-il non plus aux souvenirs eux-mêmes, mais bien au spectacle qu'on s'en donne».17 La multiplicité des récits dans les Fous de Bassan permet de vivre le roman comme une pièce de théâtre dans laquelle le même acte serait répété plusieurs fois, pièce que le spectateur verrait à chaque fois d'une perspective autre - souvent depuis divers lieux des coulisses. Grâce enfin à la dernière lettre de Stevens Brown (abolie la distance qui avait si longtemps tenu à l'écart, hors du texte, la scène du meurtre), le spectateur-lecteur verrait la pièce du point de vue de l'assassin lui-même, comme si le lecteur «y était», sur la scène même (pp. 242-249).

16. Gabrielle Poulin, «L'écriture enchantée: les Fous de Bassan d'Anne Hébert», Lettres québécoises, no 28 , Hiver $82-83$, p. 17.

17. R. Harvey, op. cit., p. 64. 
Rarement, toutefois, voit-il les choses «d'en haut», d'au-dessus de la scène: ce roman comporte peu souvent des interventions de la part d'un narrateur omniscient, hétérodiégétique. Ce qui frappe, outre la multiplicité des points de vue, c'est une pluralité «intra-narrateur». Par là, nous entendons que chaque personnage-narrateur, s'exprimant le plus souvent comme narrateur autodiégétique, décrit ou analyse parfois un autre personnage en focalisation interne, s'exprimant alors comme s'il était un narrateur hétérodiégétique. C'est le cas, par exemple, de Stevens Brown, qui décrit et raconte d'abord tout le village, et ensuite plusieurs personnages de l'extérieur, mais è proférant des réflexions sur les pensées et sentiments intérieurs des autres personnages, comme s'il était le narrateur omniscient traditionnel. Persuadé de tout savoir de la vie intérieure des personnages féminins, Stevens s'exprime parfois commè s'il savait ce que pense ou ressent son frère Perceval; sa propre victoire sur son père culmine en la capacité de connaître, à l'insu de celui-ci, sa vie intérieure: sa colère, et sa peur (p. 94). L'omniscience que s'attribue Stevens, qui voit juste souvent mais pas toujours (en témoigne sa compréhension incomplète de la psychologie de Nora et d'Olivia), est en conformité avec sa personnalité vaniteuse, son sexisme outrancier.

Le début de chaque «livre», en fait, et le début de segments moins longs, tels que le premier paragraphe (plus ou moins) de nombreuses lettres de Stevens, paraissent être rédigés en narration hétérodiégétique par un narrateur omniscient. $R$. Harvey a bien décelé un phénomène semblable à la première page de Kamouraska.18 Dans les Fous de Bassan, toutefois, rien n'interdit de lire ces passages comme étant le fait d'un narrateur autodiégétique qui livre au narrataire une vue générale du monde dans lequel il se trouve, avant d'aborder des événements l'ayant impliqué, ou sa manière de vivre ce monde, ce qui amènera l'emploi du «je». Ailleurs, c'est le dédoublement du personnage, procédé fréquent chez Anne Hébert, qui produit des passages semblant relever d'une narration hétérodiégétique, lorsque $\mathbf{N}$. Jones ou Olivia parlent d'eux-mêmes à la troisième personne, se regardant de l'extérieur. Ces cas sont à rattacher, en fait, au point de vue du personnage-narrateur se distanciant de lui-même, personnage-actant.

Seules les pages 144 et 145 semblent constituer un exemple significatif de narration hétérodiégétique. Proches apparemment de l'hétérodiégétique, les passages du «Livre de Perceval Brown et de quelques autres» relevant d'une sorte de narrateur collectif («Nous, les gens de Griffin Creek», etc.) sont, par là même, à distinguer de l'hétérodiégétique: lisons-les, toutefois, comme relevant du phénomène de la voix; car ce «nous», plus qu'il ne voit, parle, se tait - et parle pour se taire. Pour dire le faux, et pour enfermer dans le non-dit, muette, la voix du vrai. 
La première voix que nous étudierons est celle de ces «quelques autres», "gens de Griffin Creek», descendants de Loyalistes, puritains veillant à défendre leur réputation et les apparences de la respectabilité. $\mathrm{Ce}$ "nous» fait sa première apparition dès la ligne 3 du "Livre du révérend Nicolas Jones». À cela, rien d'étonnant: c'est N. Jones lui-même qui, ici, parle au nom de la tribu, dont il a bien le sentiment d'être le représentant principal (puisque chef spirituel), voire d'en constituer l'essence. Tout en restant rattaché au livre et à la voix de $\mathrm{N}$. Jones, la deuxième série d'occurrences du «nous» permet à celui-ci de mieux s'affirmer en tant que voix collective, puisqu'il s'agit d'une série de quatre «nos» (p. 26). Le pronom s'est mué en adjectif possessif, comme pour mieux prendre possession de cette voix, la libérer de l'emprise tutélaire du révérend. 19 Bientôt, le «nous» révélera sa véritable vocation de solidarité envers lui (eux)-même(s) «Nous sommes ensemble, liés les uns aux autres, pour le meilleur et pour le pire» (p. 48). Le "nous» se tait ensuite longtemps, pour ne réapparaître qu'au sein du «Livre de Perceval Brown et de quelques autres» (p. 153). D'abord occupé à raconter les recherches pour retrouver Nora et Olivia, le «nous» ne tardera pas à porter toute son attention sur l'angoisse de la culpabilité:

Des rumeurs jusque dans nos maisons, chuchotées le plus tard possible dans la nuit, lorsque nous sommes entre grandes personnes, les enfants couchés et les policiers retirés dans leur hôtel. Refaisons pour notre propre compte l'emploi du temps de tous et chacun, le soir du 31 août. (...) Celui qui nous trahira nous fera tous basculer dans le déshonneur (p. 161, p. 184).

S'élèvent aussi, dans les Fous de Bassan, d'autres voix encore. Ce roman suscite même une extension du champ de référence du vocable «voix», jusqu'ici utilisé selon la définition de Genette pour qui il renvoie à la question de savoir «qui est le narrateur?» 20 Genette précise toutefois que cette question peut être formulée autrement, pour devenir «qui parle?».21 Dans le livre d'«Olivia de la Haute Mer», Olivia est certes la narratrice de sa propre histoire (même quand elle parle d'elle-même à la troisième personne): la voix est la sienne. À sa voix pourtant, se mêle une voix autre - désignée par ce vocable (p. 217) - voix à la fois plurielle et collective, «voix de femmes» (p. 217), celle, nous dit Olivia, de «mes mère et grandmères» (pp. 216, 219, 221, 222; cf. pp. 202, 215, 217, 218).

Cette voix des femmes passées n'est pas à proprement parler une voix narrative; mais si elle ne renvoie pas à la question «Qui parle?», elle renvoie au verbe qu'utilise Genette, à «parler», puisque ces femmes "pri(e)nt»

19. Les occurrences assez fréquentes du «nous» dans «Le livre de Perceval Brown et de quelques autres» renvoient non pas à Nicolas Jones mais à un locuteur pluriel - «Nous les gens de Griffin Creek» (p. 157).

20. G. Genette, op. cit., p. 203.

21. Loc. cit. 
(p. 215), «disent» (p. 215), «chuchotent» (p. 216), «sifflent» (p. 217), «gémissent» (p. 220), «jurent» (p. 220), «recommandent» (pp. 219-220), «prennent (un) timbre clair» (p. 222), "déclarent» (p. 222), et "grondent» (p. 223): bref, cette voix «parle» (p. 217, nous soulignons); Olivia l'entend sans toujours l'écouter. En outre, le fait qu'elle se mêle ainsi à la voix narrative d'Olivia lui confère un statut particulier, qui n'est pas sans parenté avec le narratif: cette voix des femmes d'antan ne narre pas ce qui se passe, mais en lançant sans cesse à Olivia un avertissement - se méfier de Stevens Brown, de l'homme - cette voix rappelle implicitement ce qui s'est passé dans les rapports femmes-hommes depuis un temps immémorial (les agressions infligées à celles-là par ceux-ci). En plus, elle annonce (narre) implicitement ce qui se passera, cette nouvelle agression que sera le violmeurtre d'Olivia et de Nora par Stevens Brown.

La voix des femmes passées (à laquelle nous reviendrons) est la plus importante de ces autres voix qui s'élèvent dans ce roman, celle qui révèle le plus nettement la nécessité d'étendre le champ de référence du terme «voix». On ne peut guère utiliser un terme autre, puisque c'est bien le vocable qu'utilise, on l'a vu, le texte des Fous de Bassan. Ces autres voix sont caractérisées par le fait que leur intérêt réside moins en leur rattachement à tel personnage-narrateur (rattachement important néanmoins, on le verra), qu'en ce qu'elles disent, le message qu'elles énoncent. Ces voix plurielles, portées par les voix et actes de divers personnages, peuvent d'autant plus facilement prendre texte grâce à la pluralité des instances narratives.

La première de ces voix est celle du désir. Omniprésente celle-ci, ou presque: chacun des six livres, chacun des narrateurs (à l'exception du narrateur collectif, le «nous»), parle obsessionnellement du désir. Tous les personnages, narrateurs et actants, sont hantés par le désir, depuis le révérend Jones, encore sensible en 1982 au souvenir des jeunes corps de ses nièces qui l'avaient tant séduit quarante-six ans plus tôt, jusqu'au vieux Stevens Brown qui sè sent encore poursuivi par le désir des cousines Nora et Olivia, lui aussi en 1982, et que l'on devine, à la fin de sa dernière lettre, toujours aussi obsédé par le mystère du désir et de l'amour chez la femme, toujours aussi incapable de voir et de vivre la femme comme un être humain, qu'il l'était le soir du double meurtre (p. 248). En passant par les voix de Nora qui ne fait nul mystère de son désir pour Stevens - «je suis creuse et humide. En attente» - et celle d'Olivia qui reconnaît et parfois semble éprouver encore ce désir au-delà de la mort même (pp. 221-223). Quant à Perceval, G. Poulin a bien parlé de celui «dont le désir dépasse les limites et la mesure du langage et ne sait éclater qu'en cris insoutenables».22 J. Paterson a vu dans les Enfants du Sabbat une «écriture de la jouissance»:23 l'écriture des Fous de Bassan est bien plutôt celle d'un désir qui ne connaîtra guère l'assouvissement de la jouissance.

22. G. Poulin, op. cit., p. 17.

23. Janet Paterson, "L'écriture de la jouissance dans l'oeuvre romanesque d'Anne Hébert», Revue de l'Université d'Ottawa, 50, 1980, pp. 69-73. 
Le désir de Stevens Brown est celui d'un phallocrate indépassable. La deuxième voix à évoquer, dans cette énumération des «voix autres», est bien celle du machisme. Stevens vit mal son propre désir, pour lui phénomène pervers attribuable à la femme: «Une telle excitation dans tout mon corps, une rage inexplicable. Il y a trop de femmes dans ce village, trop de femmes en chaleur et d'enfants perverses» $(p, 80)$. Son irritation face à la réserve d'Olivia n'est que la pointe de l'iceberg, la synecdoque de sa misogynie:

Cette fille n'est qu'une hypocrite. (...) Les démasquer toutes. Leur faire sortir l'unique vérité de leur petit derrière prétentieux. Débarrassées des oripeaux, réduites au seul désir, humides et chaudes, les aligner devant soi, en un seul troupeau bêlant (p. 82).

«Réduites au seul désir» - et la vérité, pourtant, est que Stevens voudrait dénier aux femmes le droit de vivre leur désir:

Je la (Nora) refuse avec autant de véhémence qu'elle me désire. Épreuve de force. Il en a toujours été ainsi, je crois, chaque fois qu'une fille me fait des avances. Il faudrait les mettre au pas, toutes. Le dressage de cette vieille Maureen est déjà commencé. Je la fais de plus en plus jeûner (sexuellement). Je lui dis qu'elle est vieille et que je la quitterai bientôt (p. 90).

Stevens Jones, narrateur revivant le passé à l'aide du présent grammatical, ne semble pas se démarquer par rapport aux valeurs du violeur-assassin qu'il fut (cf. cette phrase à propos de Nora et d'Olivia: «Et moi tout seul pour les remettre à leur place»; p. 239), nonobstant les troubles psychiques que lui ont laissés ses actes (p. 248).

Stevens n'est point le seul machiste de ce roman. Son oncle, le révérend Jones, lui aussi vit toujours, en 1982, en sexiste impénitent. Pat et Pam, jumelles, soeurs de Stevens, depuis toujours, semble-t-il, interdites de séjour dans leur propre désir, en ont été réduites à n'être que les choses du vieux Nicolas Jones (p. 18). Sa femme Irène n'avait pas un statut plus élevé à ses yeux, et fut poussée au suicide par la découverte du caractère volage du désir de son mari. Même Perceval, enfin, y ira de sa tentative de viol (p. 117).

À cette voix du machisme véhiculé par des personnages masculins répondent, inlassables, des voix féminines qui sont bien, aussi, des voix féministes. Certes, il s'agit d'un féminisme peu conscient de lui-même, mais non moins réel pour autant. Felicity Jones (vénérée même par son fils Nicolas et par son petit-fils Stevens) l'a elle-même inauguré. Certes, sans cesse trompée, Felicity subit elle aussi la phallocratie. Elle s'est néanmoins réservé une heure à elle, au petit jour. Lorsque le temps le permet, elle se rend sur la grève, nage, rêve, jouit de la beauté du monde et de l'harmonie entre l'être, le corps et l'univers (pp. 34-35). Elle y amènera ses petites filles, Nora et Olivia (pp. 112-113), mais n'a point envie d'y emmener son fils Nicolas (p. 36). Celui-ci affirme que Felicity a toujours préféré les filles (p. 37), et Stevens le soutient dans cette conviction (p. 75). Les petites filles, 
pour qui «son contentement n'a pas de borne» (p. 37), feront preuve à leur tour d'une certaine affirmation des droits de la femme.

Nora, quinze ans: c'est l'affirmation et la question, la certitude de la splendeur du monde et de sa propre joie de fille qui «habite le soleil comme une seconde peau» (p. 111), l'interrogation parfois angoissée du mystère du désir et de l'amour. Etonnante ressemblance, jusque dans sa rousseur, avec la jeune Élisabeth d'Aulnières qui, elle aussi, joue dans l'eau le matin de très bonne heure, qui, elle aussi, baigne dans un monde naturel de terre et d'eau, sensoriel, riche et vivifiant, ${ }^{24}$ qui, elle aussi. (à qui l'on cache toute vérité sur la sexualité), est obligée de demander, dans l'angoisse de l'ignorance: "Comment faire? Je voudrais savoir... Les garçons... Les garçons...».25 Nora revendique le droit de désirer et d'exprimer son désir à égalité avec l'homme (p. 127). Cette revendication de l'égalité va jusqu'au plus profond, remettant en question les affirmations bibliques sur les origines. Cela est d'autant plus remarquable que remettre en question le livre de la Genèse ne doit pas être facile, dans cette tribu où l'oncle Nicolas, d'une voix autoritaire, prêche la Bible chaque dimanche:

Ce n'est pas pour rien que je joue si souvent au bord de la mer. J'y suis née (...) Faite du limon de la terre, comme Adam, et non sortie d'entre les côtés sèches d'Adam, première comme Adam je suis moi, Nora Atkins (...) avide de toute connaissance terrestre et marine (p. 116).

Nora tombe amoureuse de Stevens certes, et ses rapports avec Olivia en sont quelque peu atteints (pp. 121-122). Le problème, toutefois, résidera dans la rencontre du désir de Nora, prête à aimer d'une façon totale, physique et psychique, avec le désir malade de Stevens, qui refuse de voir en la femme un être humain, pour qui la femme est un désir qu'il méprise. Nora finira par deviner ce qu'a de pervers l'idée que Stevens se fait de la femme: «Je prétends que Stevens n'aime pas les femmes mais seulement la cochonnerie qu'on peut faire avec les femmes » (p. 130). Le mot «cochonnerie» traduit une évolution chez Nora qui trouvait tout naturel, naguère, son désir de jeune fille «en attente» (p. 118). Cette évolution est sans doute due aux expériences de Nora avec Stevens et avec le révérend Jones qui, surpris en train de lui caresser les seins, dit à Nora qu'elle est mauvaise et que par elle le péché est entré à Griffin Creek (pp. 128-129). Par la phrase «Moi aussi j'ai été cochonne avec le pasteur» (p. 130), Nora confirme que le processus par lequel l'homme culpabilise le désir féminin l'a désormais atteinte. 26

24. A. Hébert, Kamouraska, éd. cit., pp. 53, 66.

25. Ibid., p. 65.

26. Ce processus de l'envahissement progressif du personnage enfantin et adolescent par les valeurs négatives du monde adulte est fréquent dans l'oeuvre hébertienne, comme nous avons eu l'occasion de le montrer dans notre Thématique de l'enfance dans l'oeuvre poétique et romanesque d'Anne Hébert (thèse de doctorat, Université de Provence I, Aix-enProvence, 1977). 
Le lecteur retiendra surtout, néanmoins, que Nora a élevé le désir féminin au statut d'égalité avec ce Dieu mâle qu'ont créé les hommes et que prêche son oncle: «Et le Verbe s'est fait chair et Il a habité parmi nous. Et moi aussi, Nora Atkins, je me suis faite chair (...)» (p. 118). Son message final consiste à prôner le droit de la femme à vivre comme être humain entier et heureux:

Mon Dieu donnez-moi bien vite un garçon de mon âge (...) Pour le fun de tout mon corps, né pour cela, de la tête aux pieds, pour l'amour de toute mon âme, née pour cela, dans sa sauvage innocence (p. 131).

La voix d'Olivia exprime des revendications similaires. Elle devine bien que le désir d'elle chez Stevens la nie en tant qu'être humain, la réduit au statut d'objet sexuel. Mais la voix du féminin-féministe, dans le livre d'Olivia de la Haute Mer, prend la forme particulièrement intéressante de la voix des «mère et grand-mères». Cette voix dit d'abord le refus, par des femmes, de la tendance sadique des hommes à leur endroit, cette attitude sexiste si manifeste chez Stevens:

Cet homme est mauvais. Il ne désire rien tant que de réveiller la plus profonde épouvante en moi pour s'en repaître comme d'une merveille. La plus profonde, ancienne épouvante qui n'est plus tout à fait la mienne, mais celle de ma mère enceinte de moi et de ma grand-mère qui... (p. 202).

Ce passage suit de près l'évocation d'une première tentative de viol par Stevens (p. 202). La voix des femmes passées s'étend pour ne plus se limiter à la mère et la grand-mère; et ce que dira cette voix prendra toujours plus nettement la forme d'un avertissement. Ainsi, quand Stevens vient pour la première fois dire son désir à Olivia chez elle, la jeune fille, sensibilisée au danger qu'il représente, "Se raccroche le plus longtemps possible aux mouvements précis du fer sur le linge humide, une cohorte de femmes dans l'ombre et le vent la priant de continuer à repasser comme si de rien n'était»" (p. 215). Lors de la «barn dance», dira Olivia, «Mes mère et grand-mères me recommandent tout bas de ne pas lever les yeux vers lui» (p. 21.9).

Cette voix des femmes passées est d'autant plus à remarquer qu'elle a (tout comme les valeurs qu'elle exprime) ses devancières dans l'oeuvre d'Anne Hébert. Songeons à cette étonnante «femme noire, vivante, datant d'une époque reculée et sauvage» dans Kamouraska, qui, déterrée, fait peur car «Chacun se dit que la faim de vivre de cette femme, enterrée vive, il y a si longtemps, doit être si féroce et entière, accumulée sous la terre, depuis des siècles!»27 Femme dont la voix implorera le droit de vivre - mais à qui tous fermeront leur porte, la laissant mourir de faim et de solitude. ${ }^{28}$ Cette "faim de vivre», ce désir de vie et d'amour si fort chez Nora et Olivia n'a

27. Anne Hébert, Kamouraska, éd. cit., p. 250

28. Loc. cit. 
point, dans le monde qu'habite ces femmes, le droit de s'exprimer. L'image de la femme noire dans Kamouraska porte un avertissement: celle qui se permet d'exprimer sa faim de vivre risque, dans l'univers romanesque de Kamouraska comme dans celui des Fous de Bassan, d'être châtiée à l'extrême. Message semblable à celui qu'adresse à Olivia ses «mère et grandmères»: le malheur frappe Olivia à partir du moment où elle cesse de se méfier de Stevens Brown, lui donne le bras, accepte de descendre à la plage avec lui. Dans les Enfants du Sabbat la mère de Julie Labrosse lui a dit: «Tu es ma fille et tu me continues"; 29 chez cette mère, comme chez Julie, le statut de sorcière a quelque chose de quasi héréditaire; elles font partie d'une longue lignée de sorcières remontant (au moins) jusqu'à «Barbe Hallé, née vers 1645, à La Coudray, en Beauce, France». ${ }^{30}$ Soeur Julie, en revêtant à son tour la vocation de sorcière; obéit à la voix de ses propres mère et grand-mères, voix qui lui propose un mode de vie - la sorcellerie qui comportait dès le début de la lignée, le texte romanesque le souligne, l'opposition au mâle: le mari de Barbe Hallé «n'a jamais pu 'ménager' avec elle parce qu'elle était une sorcière». ${ }^{31}$

Cette vocation que propose cette voix des femmes passées est donc quelque chose qui remet en question la distribution des rôles et du pouvoir entre hommes et femmes et qui traduit une méfiance chez la femme envers la domination que les hommes voudraient exercer sur elle. Voilà que l'on retrouve ainsi un message bien voisin de celui dont sont porteuses les voix des mère et grand-mères d'Olivia.

Anne Hẻbert a signalé la présence novatrice, dans nombre d'écritures de femmes contemporaines, d'une «voix féminine» empreinte de force, et a précisé que «l'écriture des femmes explore des mémoires qui sont souterraines parce qu'elles ont été enfouies assez longtemps. Olivia est très sensible justement à toutes ces voix de femmes qui l'ont précédée, ces mères et ces grand-mères». ${ }^{32}$ La grand-mère Felicity Jones non seulement revendique une vie à elle, un temps du féminin (les bains de mer à l'aube) mais aussi dans la mesure où ce rite, avec sa dimension «rapprochement avec les grandes forces de la nature, avec la mer et la terre" a quelque chose du rite païen - Felicity remet en cause le pouvoir des hommes, pouvoir qui ne s'est nulle part mieux incarné que dans le type de religion phallocrate que prêche Nicolas Jones. Cette religion-là, la jeune Nora la subvertit à son tour en revendiquant son égalité avec l'homme depuis les origines du monde:

Ce n'est pas pour rien que je joue si souvent au bord de la mer. J'y suis née (...) Faite du limon de la terre, comme Adam, et non sortie d'entre les côtés sèches d'Adam, première comme Adam (...) (p. 116; nous soulignons).

29. Anne Hébert, les Enfants du Sabbat (roman), Paris, Ed. du Seuil, 1975, p. 125.

30. Ibid., pp. 103-104.

31. Ibid., p. 104.

32. Anne Hébert, entretien avec Jean Royer, le Devoir, 11 décembre 1982, p. 21. 
Le statut d'avertissement - et donc de message que revêt ce que dit la voix des femmes passées - implique une extension encore plus grande du champ que recouvre le vocable "voix» ici; car énoncer un avertissement pareil, qui vise à protéger une femme (la femme) contre un homme (l'homme), et qui exhorte les femmes à rester entre elles, en une solidarité féminine, c'est énoncer un message relevant d'une idéologie de caractère féministe; entendons par là: qui valorise la femme (en outre dans ce roman, la femme constitue le bien, les personnages masculins représentent le mal):

Si la notion, qu'exprime G. Poulin, de «voix multiple», de "voix multiforme» 33 nous paraît juste, il nous semble plus exact de voir cette multiplicité non en la mer et en sa/ses voix, mais comme prenant texte de façon précise dans les différents personnages-narrateurs et surtout dans ces "voix autres» qui tissent dans le roman tout un jeu de rapports entre le désir, le masculin/machiste, et le féminin/féministe. Certes, la voix du désir procède en partie des deux autres, l'opposition masculin-féminin, machisme-féminisme se fondant largement sur une opposition entre un désir masculin égoïste, malsain, découlant de pulsions destructrices, orienté vers la mort; et un désir féminin sain, positif, tourné vers la vie et le bonheur. ${ }^{34}$

Pourtant, dans ce choc de deux désirs, le féminin et le masculin, la force (mais non point le droit) paraît être du côté de celui-ci, et le dénouement du 31 août 1936 donne à la vision hébertienne de cette lutte un caractère pessimiste rappelant celui de la dernière page de Kamouraska (échec des tentatives de la femme noire et d'Élisabeth pour conquérir la liberté et le bonheur), voire celle des Enfants du Sabbat. ${ }^{35}$ Pessimisme? À moins que l'espoir ne réside dans le fait même de la révolte - et dans le fait même que celle qui écrit la chronique de cette longue lutte, celle qui a non seulement «reçu» mais su assumer la «fonction de la parole» ${ }^{36}$ soit une femme. Ainsi s'aperçoit-on de la pertinence du point de vue et de la voix de l'auteure, et de la distance qu'elle met ou ne met pas entre elle-même et la lutte qu'elle met en texte. Or, il nous semble bien que, par le fait même d'écrire cette lutte, la romancière réduit cette distance à zéro. Est là, présente. Dans la lutte, de par l'acte même de l'écrire.

33. G. Poulin, op. cit, pp. 16, 18.

34. La veuve Maureen semble être fortement influencée par la conception de la femme comme être relatif: Olivia le sent bien, qui décrit Maureen comme étant «pareille à quelqu'un qui attend furieusement une grâce improbable» (p. 201), grâce qu'elle attend de Stevens Brown qui l'a prise pour ensuite la rejeter et la mépriser. Cette vision de Maureen est à prendre sous réserve toutefois, car ce personnage nous est présenté principalement à travers les yeux et le récit d'un personnage-narrateur masculin et machiste - Stevens Brown. Le personnage de la veuve Maureen permet aux Fous de Bassan de mettre en relief la condition féminine dans cet unjvers romanesque - condition d'être relatif et de victime - ainsi que le machisme de Stevens Brown.

35. Voir, à propos de la fin des Enfants du Sabbat, notre "les Enfants du Sabbat et la problématique de la libération chez Anne Hébert", Études canadiennes, Talence, Association française d'études canadiennes, 1980, pp. 41-42.

36. Anne Hébert, «Mystère de la Parole», Poèmes, Paris, Ed. du Seuil, 1960, p. 75. 
L'étude des phénomènes narratifs que sont la distance, le point de vue et la voix permet ainsi de mieux comprendre le "comment", le fonctionnement d'une écriture romanesque d'une qualité remarquable. Le fait d'avoir écouté, parmi et en plus des "voix» au sens purement narratologique, ces "voix autres» que sont celles du désir, du masculin/machiste et du féminin/ féministe nous a permis de repérer un aspect de l'oeuvre hébertienne dont on ne prend pas toujours conscience, et qui est l'articulation, dans'cette oeuvre, du scriptural et de l'idéologique. Articulation, donc, de la recherche dans l'art d'écrire, d'une part, et, de l'autre, d'une idéologie de la libération et de l'épanouissement des femmes. 